PharmacoEconomics \& Outcomes News 865, p23 - 31 Oct 2020

\title{
Linked HIV/SARS-CoV-2 testing could reduce incidence of HIV and costs
}

Implementing linked HIV testing alongside SARS-CoV-2 testing has the potential to reduce the number of HIV infections substantially and reduce direct and indirect healthcare costs attributable to HIV.

This is the main finding of a study that used an HIV transmission model to assess the potential impact of widespread, linked, opt-out HIV testing combined with SARS-CoV-2 testing on the HIV/AIDS epidemics in six US cities (Atlanta, Baltimore, Los Angeles, Miami, New York City and Seattle). The cost effectiveness of this strategy was also assessed, accounting for a range of hypothesised effects of COVID-19 on HIV risk behaviours and access to treatment and prevention services.

The model predicted that linked, opt-out HIV testing alongside SARS-CoV-testing and contact tracing could reduce HIV infections between 2020-2025 in all six cities, ranging from 576-696 (1.6\%-1.7\%) fewer infections with 10\% offered HIV testing (assuming 6.6\% accepting a test) up to 5840-7225 (16.3\%-17.2\%) fewer infections with 90\% offered testing (assuming 59.3\% accepting a test). The initial incremental upfront investment required for the HIV testing strategy was estimated at between \$US20.6 million to \$220.7 million across cities (year 2018 values). However, in the long term, the intervention would result in savings in healthcare costs across all cities. "Populationlevel SARS-CoV-2 viral and serological testing may provide a unique opportunity to conduct HIV testing, among other health promotion activities," suggest the researchers.

Zang X, et al. The potential epidemiological impact of COVID-19 on the HIV/AIDS epidemic and the cost-effectiveness of linked, opt-out HIV testing: A modeling study in six US cities. Clinical Infectious Diseases : 12 Oct 2020. Available from: URL: http://doi.org/10.1093/cid/ciaa1547 